\title{
What's new in IL-12? Combination!
}

\author{
Chris E Lawrence ${ }^{1 *}$, Mark Rubinstein², Zoya Gluzman-Poltorak', Vladimir Vainstein ${ }^{1}$, Lena A Basile \\ From 30th Annual Meeting and Associated Programs of the Society for Immunotherapy of Cancer (SITC 2015) \\ National Harbor, MD, USA. 4-8 November 2015
}

IL-12 is a heterodimeric, pro-inflammatory cytokine that enhances the cytotoxic activity of natural killer (NK) cells and cytotoxic $\mathrm{CD}^{+} \mathrm{T}$-lymphocytes, and induces an IFN$\alpha$-dominated Th1 CD4 $4^{+}$T-lymphocyte response. IL-12 as an immunotherapeutic agent administered subcutaneously in cancer patients has demonstrated clinical responses in melanoma, T cell lymphoma, non-Hodgkin's lymphoma, and AIDS-related Kaposi sarcoma, but was never developed further. Having elucidated novel hematological properties of IL-12, we are advancing our proprietary recombinant human IL-12 (NM-IL-12) for the treatment of the Hematopoietic Syndrome of Acute Radiation Syndrome. In three clinical safety studies conducted in over 200 healthy human volunteers, subcutaneous NM-IL12 was well-tolerated. No adverse immune reactions or immunogenicity were observed.

We have now developed a novel clinical paradigm for the use of subcutaneously administered, low dose NMIL-12 combined with standard of care radiotherapy, chemotherapy, or immunotherapy for the treatment of cancer. The pleiotropic effects of IL-12 are expected to augment the mechanistic, anti-tumor effects of each of these treatments.

In vitro NM-IL-12 stimulated primary human NK cell secretion of IFN-g and the cytotoxic lysis of leukemic cells, and inhibited production of pro-angiogenic IL-17 in human peripheral blood mononuclear cells. In vivo, recombinant murine IL-12 (rMuIL-12) caused significant tumor growth inhibition following total body irradiation (625cGy) in syngeneic Lewis lung and EL4 lymphoma tumor models. In the same models, rMuIL-12 in combination with cyclophosphamide also caused significant tumor growth inhibition. In the case of the non-immunogenic Lewis lung cancer model the combination of chemotherapy and IL-12 enhanced immunogenicity. In both tumor models, the antitumor effects of IL-12 were accompanied by rapid recovery of neutrophils, platelets and red blood cells, depressed by radiation or chemotherapy. This suggests an additional benefit of NM-IL-12 to cancer patients myelosuppressed following radiation or chemotherapy.

Preclinical evaluation of NM-IL-12 with radiation therapy and chemotherapy is now followed by evaluating combination immunotherapy. PD-1 blockade elicits potent anti-tumor immunity in a subset of melanoma patients. We have thus evaluated the combination of rMuIL-12 and anti-PD-1 antibody in a clinically relevant, syngeneic model of spontaneous, highly metastatic B16 mouse melanoma has been tested.

In summary we show that NM-IL-12 has excellent anti-tumor potential when used preclinically in combination with standard of care anti-cancer treatments, including radiation, chemotherapy and immunotherapy. NM-IL-12 is expected to contribute durable anti-tumor responses in the clinic through potent immunoactivation and anti-angiogenic effects, and to replenish blood cells, while being safe, well tolerated and non-immunogenic.

\section{Authors' details \\ ${ }^{1}$ Neumedicines, Pasadena, CA, USA. ${ }^{2}$ Medical University of South Carolina, Charleston, SC, USA.}

Published: 4 November 2015

doi:10.1186/2051-1426-3-S2-P363

Cite this article as: Lawrence et al.: What's new in IL-12? Combination! Journal for ImmunoTherapy of Cancer 2015 3(Suppl 2):P363.

${ }^{1}$ Neumedicines, Pasadena, CA, USA

Full list of author information is available at the end of the article

(c) 2015 Lawrence et al. This is an Open Access article distributed under the terms of the Creative Commons Attribution License (http:// creativecommons.org/licenses/by/4.0), which permits unrestricted use, distribution, and reproduction in any medium, provided the original work is properly cited. The Creative Commons Public Domain Dedication waiver (http://creativecommons.org/publicdomain/ zero/1.0/) applies to the data made available in this article, unless otherwise stated. 